\title{
EXOSAT OBSERVATIONS OF THE SUPERNOVA REMNANT CAS A
}

F A Jansen. S P McKechnie. P A. J. de Korte,

J A. M. Bleeker. E Gronenschild. “ A. Peacock. '

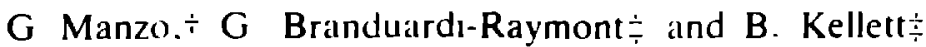

'Laboraron for Space Research Leiden, PO Box 9504,

Huygens Laboratormum. Leiden. The Nesherlands

* Laboratury for Space Research Unechr. L'rrecht. The

Netherlands

**r Space Science Deparmem of ESA. ESTEC, Noordwilk. The

Netherlands

- Isturulo di Fisica dell Liniersia Palermo, Palermo, Itah!

†ullard Space Science Laboratory, Holmbur Si Mary, U.K

ABSTRACT

The young supernova remnant Cas A has been observed with the imaging proportional counter (PSD) of the EXOSAT observatory. High quality spatially resolved, spectral data allow for the first time the determination of the temperature structure of the remnant.

Preliminary results on the distribution of temperature and emission measure over the remnant are presented.

\section{INTRODUCTION}

Although the nature of Cas $A$ as a type II supernova event has been questioned $/ 4 /$, it 15 quite certain that 1 ts progenitor was a massive star (up to $24 \mathrm{M}_{\odot}$ has been guggested /1/ that exploded in a inhomogeneous medium approximately 400 years ago. Current knowledge of the $X$-ray status of the remnant is primarily based on several papers describing its observation wh the EINSTEIN observatory $/ 11,2,9 /$.

The SSS data were fitted with isothermal plasma models in collisional equilibrium. Adequate fits could be obtained for a two temperature model with $k T=0.63$ and $4 \mathrm{keV}$, assuming an $\mathrm{N}_{h}$ of $1.5 * 10^{22} \mathrm{~cm}^{-2}$ and over-abundances of certain elements $11 \mathrm{ke} \mathrm{si}_{1}$ and $\mathrm{s} / 2 \%$.

The IPC data in particular, suqgested a temperature varıation over the remnant, this follows from the hardness ratio plot made over radial annuli /11/. These data clearly indicate the need for better, spatially resolved, spectral data, whıch are now avallable from the ExOSAT PSD.

OBSERVATION AND DATA ANALYSIS.

The supernova remnant Cas A has been observed for fourteen hours with the Imaging proportional counter (PSD) of the EXOSAT observatory. With this instrument spatially resolved $(1$ arcmin.). spectral data (E/ E -2.5 at $1 \mathrm{keV} / 5 /$ ) of good statistical quality (typically several thousand cts. per analysis bin) have been obtained.

One of the key problems in analysing the spectral dasa in the 0.3-2.4 keV band as obtained by the position sensitive detector is the correlation between several spectral parameters. Especially the strength of the Fe-L line complex between 0.5 and 1 kev, as a derived parameter of $\mathrm{Fe}$ abundance and plasma cemperature, $1 \mathrm{~s}$ strongly anti-correlated with the spectral cut of caused by interstellar absorption $/ 2 \%$. Therefore the data will not allow for a determination of both temperature. Fe-abundance and interstellar column dengity independently. This led us to decide to use the interstellar colum density as derived from other data sets in order to be able to determine the cemperature structure of the remnant.

The hydrogen column density derived from $21 \mathrm{~cm}$ radio observations enables us to correct for the interstellar absorption. Since cas A 15 located behind the perseus arm of our galaxy the hydrogen column density is expected to change on a scale size much smaller then the size of the remnant $/ 6 /$.

The total $\mathrm{N}_{h}$ value along the line of sight towards Cas A is made up of two components. One is constant and represents the hydrogen column density between the earth and the perseus arm and is almogt constant, the other shows considerable variation and is due to granularity of the gas in the perseus arm. We used the 21 om optical depth measurements /e/,obtained with a resolution of 1 arc minute, to assess this second, spatially variant component. The total $\mathrm{N}_{h}$ values thus obtained were weighted with the number of $x$-ray counts to get $N_{h}$ values for the 16 elements in which we analysed the remnant (see fig. 1). The resulting $N_{h}$ values are summarized in table 1 . 


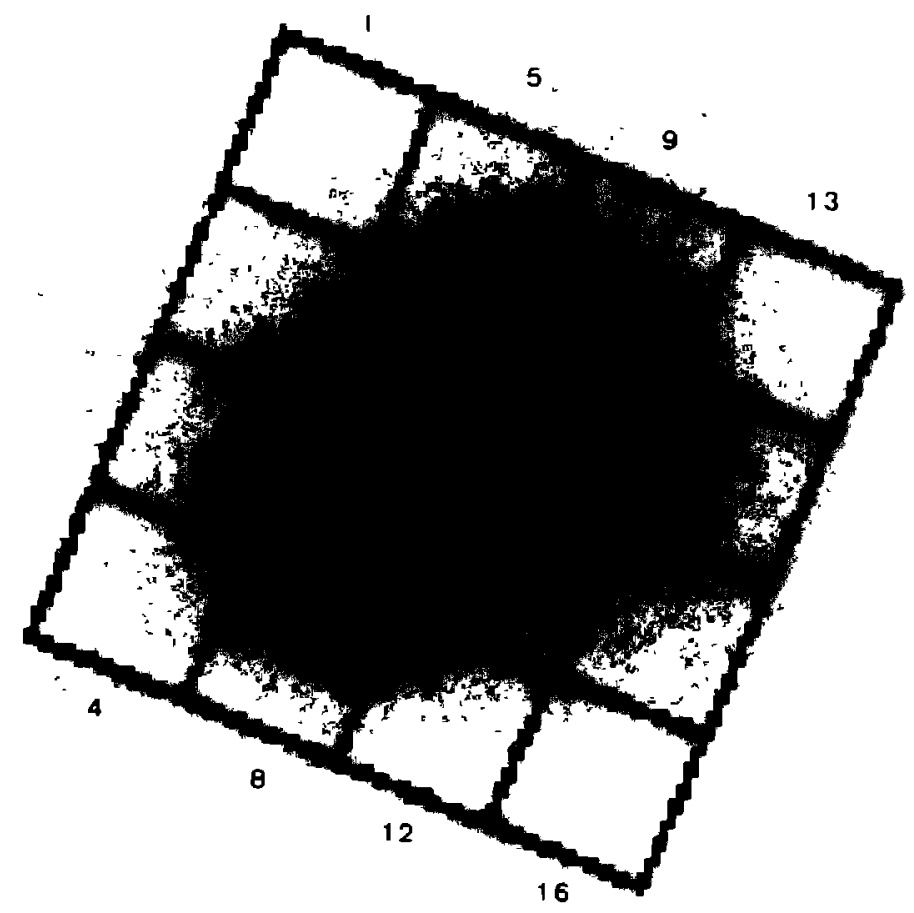

Fig. 1 The PSD image of Cas A. Qverlaid are the 16 numbered elements in which the remnant was analysed. These numbers correspond to colum one of iable (north is up).

Table 1.

\begin{tabular}{|c|c|c|c|c|c|c|c|}
\hline Box & $\mathrm{nr}$. & $\begin{array}{l}\text { Hean RA } \\
H H: M M: S S\end{array}$ & $\begin{array}{l}\text { Mean DEC } \\
\text { DD: MM: SS }\end{array}$ & $\left(10^{20^{\mathrm{N}}} \mathrm{cm}^{-2}\right)$ & $\begin{array}{l}T^{\star} \\
\left(+e^{*}\right)\end{array}$ & Counts & $\mathrm{EM}^{\star \star}$ \\
\hline & 1 & $23 \cdot 21: 19$ & $58: 45: 50$ & 133 & $0.34 \pm 7.05$ & 437 & $11) . A$ \\
\hline & 2 & $23,21: 23$ & $58: 44: 29$ & 146 & $0.42 \pm 0.05$ & 1291 & 32.7 \\
\hline & 3 & $23: 21: 28$ & $5 B: 43: 08$ & 142 & $0.4 P \pm 0.03$ & 2150 & 43.0 \\
\hline & 4 & $23: 21: 33$ & $58: 41: 27$ & 121 & $0.53 \pm 0.07$ & 919 & 11.1 \\
\hline & 5 & 23. $21: 09$ & $5 B: 45: 18$ & 150 & $0.32 \pm 0.03$ & 2380 & 74.6 \\
\hline & 6 & $23: 21: 13$ & $58: 43: 56$ & 151 & $0.45 \pm 0.01$ & 5042 & 155.0 \\
\hline & 7 & $23: 21: 17$ & $58: 42: 35$ & 152 & $0.52 \pm 0.71$ & 8584 & 188.0 \\
\hline & B & $23: 21: 22$ & $58: 40: 55$ & 145 & $0.53 \pm 0.03$ & 4267 & 79.2 \\
\hline & 9 & $23: 20: 58$ & $58: 44: 45$ & 156 & $0.43 \pm 0.03$ & 3620 & 109.3 \\
\hline & 10 & $23: 21: 02$ & $58: 43: 24$ & 154 & $0.47 \pm 0.02$ & 7018 & 178.6 \\
\hline & 11 & $23 \cdot 21: 07$ & $58: 42: 02$ & 149 & 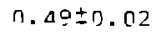 & 5196 & 112.3 \\
\hline & 12 & $23: 21 \cdot 12$ & $58: 40: 22$ & 147 & $0.51 \pm 0.03$ & $267 n$ & 51.9 \\
\hline & 13 & $23: 20: 48$ & $58: 44: 12$ & $15 R$ & $0.42 \pm 0.04$ & 1173 & 35.6 \\
\hline & 14 & $23-20: 52$ & $5 \theta: 42: 51$ & 157 & $0.59 \pm 0.03$ & 3240 & 65.5 \\
\hline & 15 & $23 \cdot 20: 56$ & $58: 41 \cdot 29$ & 153 & $0.53 \pm 0.03$ & 2552 & 52.4 \\
\hline & 16 & $23: 21: 01$ & $58: 39: 49$ & 147 & $0.50 \pm 0.05$ & 831 & 15.7 \\
\hline
\end{tabular}

- Errors are 90 confidence levels

*) Emission measure in units of $10^{57} \mathrm{~cm}^{-3}$ for a distance of $2.8 \mathrm{kpC}$

The average value of $1.5 * 10^{22} \mathrm{~cm}^{-2}$ obtained for the remant as a whole :s equal to that derived earlier /2/.For the calculatinn nt the tray absorption by hydrogen we used the most 
recent values $/ 10 /$.

To fit our data we used a plasma spectrum model /7/. In order to determine the elemental abundances needed in such a code we started out by fixing all elements but silicon to their solar abundance. This was done because the PSD with its limited spectral range and resolution (as compared to the EINSTEIN SSS) is most sensitive to the $S_{1} 1$ ines around 1.8 keV and almost insensitive to the $\mathrm{S}$ lines around $2.4 \mathrm{keV}$. The Fe abundance was not changed because of the aforementioned correlation. We thus obtained a ratio to solar abundance for 51 of $2.3 \pm 0.4$ (908 confidence). Comparing this result to the Einstein sss value for 51 of $1.7 \pm 0.1$ and considering that we use a different plasma code and hydrogen absorption for $x-r a y s$, we conclude that there is a good consigtency between our data and the Enstein data. This led us to use the Einstein abundances for all further analysis $/ 2 /$.

Therefore, using the $\mathrm{N}_{\mathrm{h}}$ values from table 1 we obtained temperatures and emission measures for all 16 elements over the remnant, these values are sumnarised in table 1 . A fit to the data for the whole remnant is shown in $\mathrm{fig} .2$.

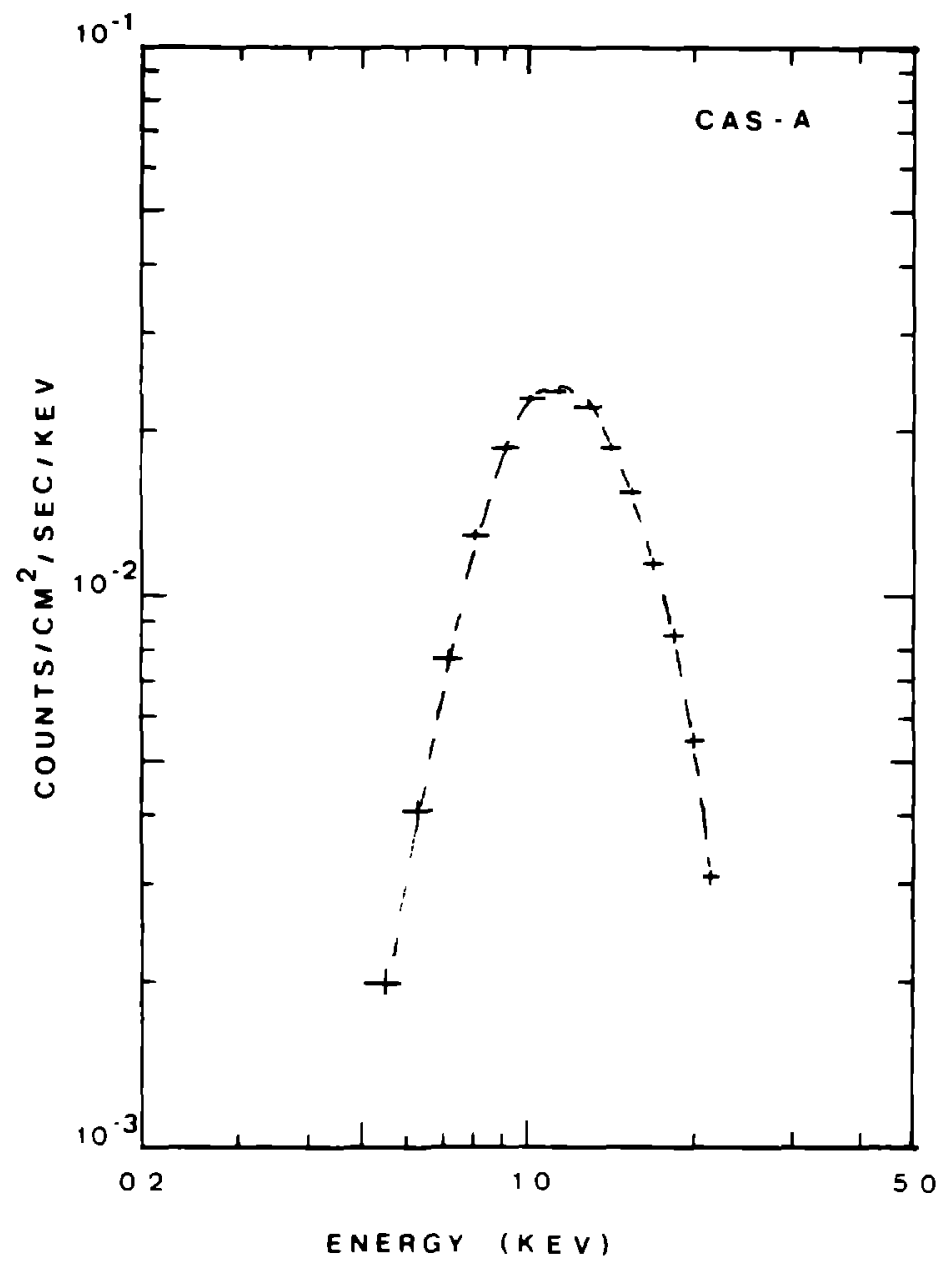

Fig. 2 The Observed spectrum of the total remnant (crosses), the dashed ilne is the optimum fit for $\langle\mathrm{kT}\rangle=0.47 \mathrm{keV}$

$$
\left\langle N_{h}\right\rangle=1.47 * 10^{22}
$$

DISCUSSION AND CONCLUSIONS

The temperatures derived from our data show a clear variation over the remnant. Assuming isothermal plasma model spectra there appears to be a trend of temperature 1 ncrease from the northern to the southern part of Cas A. However, the observed average temperature of 0.5 keV cannot explain the strength of the iron line at $6.7 \mathrm{keV}$ as it 15 observed by the ExosAT GsPC. 
It 1 obvious however that for Cas A Non Ionisation Equilibrium ('ile) rodels should be applied in view of its age. This has a influence on temperatures as well as atundances/12/. The use of NIE-models to arrive at a selfconsistent model explaining the PSD $2-d$ GSPC spectra is the subject of a forthcoming paper.

1. Arnett, W. D., 1975, Ap. J., 195, 727

2. Becker, R.H., Holt, S.S., Smith, B.W., White, N.E., Boldt, E.A., Mushotzky, R.F., Serlemitsos, P.J., 1979, Ap. J., 234, L73

3. Brown, R.L., Gould, R.J., 1970, Phys. Rev. D, 1, 2252

4. Chevalier, R.A., 1976, Ap. J., 20̄, B26

5. de Korte, P.A.J. et al. $\overline{1981}$, Spc. Sc1. Rev., 30, 495

6. Greisen, E.W., 1973, Ap. J., 184, 363

7. Gronenschild, E.H.B.M., Mewe, R., 1978, Astron. E Astrophys. Suppl., 32,283

8. Kalberla, Goss, M., Schwartz, U., 1984, In preparation

9. Markert, T.H., Canizares, C.R., Clark, G.W., 1983, A... J., 268, 134

10. Morrison, R., McCammon, D., 1983, Ap. J., 270, 119

11. Murray, S.S., Fabbiano, G., Fabian, A.C., Epstein, A., Giacconi, R., 1973, Ap. J.. 234, L69

12. Shull, J.M., 1982, Ap. J., 262, 308 